Research Article

\title{
Fentanyl Exerts an Antitumor Effect on Papillary Thyroid Cancer by Regulating the miR-204/KLF5 Axis
}

\author{
Dahao Lu, ${ }^{1}$ Lulu Jiang, ${ }^{2}$ Chen Dai, ${ }^{1}$ Keshi Yan, ${ }^{1}$ and Ju Gao $\mathbb{D}^{1}$ \\ ${ }^{1}$ Department of Anesthesiology, Northern People's Hospital, Medical College of Yangzhou University, China \\ ${ }^{2}$ Department of Anesthesiology, The Second Xiangya Hospital of Central South University, China \\ Correspondence should be addressed to Ju Gao; subei015047@163.com
}

Received 24 February 2021; Revised 1 March 2021; Accepted 8 March 2021; Published 26 March 2021

Academic Editor: Tingting Hong

Copyright (c) 2021 Dahao Lu et al. This is an open access article distributed under the Creative Commons Attribution License, which permits unrestricted use, distribution, and reproduction in any medium, provided the original work is properly cited.

\begin{abstract}
Fentanyl is a strong anesthetic analgesic drug that plays important roles in many types of cancers. However, the role of fentanyl in papillary thyroid cancer (PTC) tumor development remains ambiguous. In this study, we aimed to investigate the potential antitumor effects of fentanyl on PTC cell viability and invasion. Results of cell counting kit-8 and Transwell assays demonstrated that fentanyl treatment $(5 \mathrm{ng} / \mathrm{ml})$ reduced the viability and invasion of two PTC cells, TCP-1 and BCPAP. Our data subsequently showed that fentanyl induced antitumor effects by increasing miR-204 expressions. Furthermore, the results of luciferase reporter assays identified that miR-204 directly targets Krüppel-like transcription factor 5 (KLF5), which serves as tumor-promoting genes in many cancers. Further mechanistic analyses revealed that fentanyl performs its tumor-suppressive functions by regulating the miR-204/KLF5 axis in PTC cells. These results contribute to understanding the important role of fentanyl in treating PTC.
\end{abstract}

\section{Introduction}

As the most common endocrine-related malignant tumor over the past decades, thyroid cancer has rising incidence with an annual rate of $3 \%[1,2]$. Papillary thyroid cancer (PTC) is a common histologic type of thyroid cancer that has attained considerable attention in recent years [3]. Although the majority of patients with PTC show relatively good prognosis, some cases still suffer from poor outcomes due to local recurrence and distant metastasis [4]. Thus, determining the underlying mechanism related to PTC tumor metastasis and developing new therapeutic targets against PTC are crucial.

Fentanyl is a strong anesthetic analgesic drug that has been widely applied in clinical anesthesia, especially during cancer surgery [5]. However, fentanyl plays not only important roles in pain management but also exerts its tumorsuppressing functions in many types of cancers [6]. Fentanyl showed an inhibitory effect on cell invasion and migration via the promotion of the miR-302b-ErbB4 axis in esophageal squamous cell carcinoma (ESCC) [7]. Li et al. [8] demonstrated that fentanyl retarded tumor progression in gastric cancer by repressing the expression of matrix metalloproteinase 9 (MMP-9).

MicroRNAs (miRNAs) with a length of nearly 22 nucleotides are a class of noncoding RNAs that perform important regulatory roles in the pathogenesis of many cancers [9-11]. miRNAs regulate their target expressions posttranscriptionally by pairing with $3^{\prime}$-untranslated region ( $3^{\prime}$ UTR) of genes [12]. Recent studies have reported the role of miR-204 in several human cancers, including osteosarcoma, gastric cancer, and acute myeloid leukemia [13-16]. Interestingly, miR-204 was found to be downregulated in papillary thyroid carcinoma tissues [17], while no further mechanistic analysis was performed. Bioinformatics analysis shows Krüppel-like transcription factor 5 (KLF5) as a putative target gene of miR-204. KLF5 has been reported to promoted PTC cell growth, invasion, and migration from previous evidence [18]. In this study, biological functions of fentanyl in PTC cancer progression and its regulatory mechanisms involving 
the miR-204/KLF5 axis in reducing cell viability and invasion are explored in this study. The findings of our work can provide new insights into the treatment of PTC.

\section{Materials and Methods}

2.1. Cells and Tissues. PTC cell lines (TPC-1 and BCPAP) were obtained from the Shanghai Institute for Biological Sciences (http://www.cellbank.org.cn/) and cultured in RPMI1640 medium (Gibco, USA) containing 10\% fetal bovine serum (FBS). Both cells were incubated at $37^{\circ} \mathrm{C}$ with $5 \%$ $\mathrm{CO}_{2}$. Fentanyl (Sigma-Aldrich, USA) was dissolved in dimethyl sulfoxide (DMSO) and added into the culture medium at different concentrations $(0,0.5,5$, and $50 \mathrm{ng} / \mathrm{ml})$. Addition of only DMSO into the culture medium was performed for the control group.

2.2. Cell Transfection. miR-204 mimic/inhibitor and negative controls (NCs) were purchased from RiboBio (Guangzhou, China). KLF5 expression vector, empty vector, si-KLF5, and scramble siRNA (si-NC) were also designed by RiboBio (Guangzhou, China). TPC-1 and BC-PAP cells were cultured in six-well plates with miR-204 mimic, NC mimic, miR-204 inhibitor, NC inhibitor, KLF5, or NC using Lipofectamine 2000 (Invitrogen, CA) for transfection according to the manufacturer's recommendations.

2.3. RNA Extraction and Quantitative Real-Time PCR Assays. cDNA was reversely transcribed from RNA using a miRNA RT-PCR kit (Takara, China) for miR-204 expression analysis. cDNA was reversely transcribed from RNA using an mRNA RT-PCR kit (Takara, China) for KLF5 expression analysis. Expression levels of miR-204 and KLF5 were quantified using SYBR Green (Applied Biosystems, USA) on an ABI 7500 Fast DX RT-PCR instrument (Applied Biosystems, USA). Primer sequences of miR-204 and U6 were synthesized by RiboBio (Guangzhou, China). Specific primers for KLF5 and $\beta$-actin used in the study are presented as follows: KLF5: $5^{\prime}$-CCTGGTCCAGACAAGATGTGA-3', $5^{\prime}$-GAAC TGGTCTACGACTGAGGC- $3^{\prime}$ and $\beta$-actin: $5^{\prime}$-GGACTT CGAGCAAGAGATGG-3', $5^{\prime}$-AGCACTGTGTTGGCGT ACAG-3' . Fold changes for miR-204 and KLF5 expressions were calculated using the $2^{-\Delta \Delta \mathrm{Ct}}$ method.

2.4. Cell Counting Kit-8 (CCK-8) Assays. CCK-8 (KeyGEN) assays were used to evaluate the viability of PTC cells. Transfected PTC cells were planted into 96-well plates at a density of $3 \times 10^{3}$ cells/well. The CCK- 8 solution $(10 \mu \mathrm{l})$ was added to cultured cells in $100 \mu \mathrm{l}$ of culture medium $24 \mathrm{~h}$ after treatment and incubated at $37^{\circ} \mathrm{C}$ with $5 \% \mathrm{CO}_{2}$ for $1 \mathrm{~h}$. Absorbance values were then determined at $450 \mathrm{~nm}$ after $1,2,3$, and 4 days.

2.5. Transwell Assays. Transwell inserts with Matrigel (BD Biosciences) were used to detect the invasion ability of PTC cells. Then, $200 \mu \mathrm{l}$ of serum-free media with a density of 2 $\times 10^{4}$ cells were transferred to the upper chamber (Corning; NY, USA). A $500 \mu \mathrm{l}$ medium containing $20 \%$ FBS was subsequently added to the lower compartment. Cells that moved to the bottom of the chamber were stained with $0.2 \%$ crystal violet after incubation for $24 \mathrm{~h}$. The number of invading cells from five independent visual fields was counted under an inverted microscope with a magnification of $\times 200$.

2.6. Western Blot Analysis. Transfected PTC cells were lysed in RIPA buffer (Beyotime; Shanghai, China) after washing with phosphate buffer solution (PBS). Extracted proteins were subsequently measured using a bicinchoninic acid (BCA) kit (Beyotime; Shanghai, China). Then, $15 \mu \mathrm{g}$ of protein from each sample was separated on $10 \%$ sodium dodecyl sulfate-polyacrylamide gel electrophoresis (SDS-PAGE) gels and transferred onto a polyvinylidene fluoride (PVDF) membrane (Miilp, USA). The membrane was incubated with rabbit polyclonal anti-KLF5 ( $1: 1000$, Abcam, USA) after blocking with $5 \%$ defatted milk at $4^{\circ} \mathrm{C}$ for $2 \mathrm{~h}$. Anti- $\beta$-actin ( $1: 2000$, CST, USA) was used as the loading control. Immunoreactive bands were visualized using an enhanced chemiluminescence reagent (Beyotime; Shanghai, China).

2.7. Luciferase Reporter Assay. We used a luciferase reporter assay to analyze the binding between miR-204 and KLF5. PTC cells cotransfected with miR-204 or NC mimic and pCDNA3.1 luciferase reporter vector containing KLF5-3' UTR wild-type (wt) vector or KLF5-3'UTR mutant (mut) fragment using lipofectamine 2000 reagents (Invitrogen; Carlsbad, CA). The Dual-Luciferase Reporter Assay System (Promega) was used to determine luciferase activities after transfection for $48 \mathrm{~h}$.

2.8. Statistical Analysis. Data were statistically analyzed by Student's $t$-test, one-way analysis of variance (ANOVA) with Tukey's test, and repeated measurement ANOVA with Bonferroni corrections using GraphPad 5.0 and SPSS 19.0, respectively. Values were shown as mean \pm standard deviation from at least three independent experiments. The $P$ value $<0.05$ was considered statistically significant.

\section{Results}

3.1. Fentanyl Reduced PTC Cell Viability and Invasion. We first used CCK- 8 assays to detect the viability of PTC cells under different concentrations of fentanyl stimulation. Figure 1(a) shows that fentanyl reduces TCP-1 and BCPAP cell viability at a dosage- and time-dependent manner. Transwell invasion assays also illustrated that TCP-1 and BCPAP cell invasion was significantly inhibited by fentanyl treatment in a dose-dependent manner (Figures 1(b) and 1(c)). These data demonstrated that the fentanyl treatment repressed the cell viability and invasion of PTC cells.

3.2. Fentanyl Repressed PTC Cell Viability and Invasion via Upregulation of miR-204. A previous study has reported that miR-204 is downregulated in PTC tissues and cells, and its overexpression antagonizes the tumorigenicity of PTC cells [17]. To the best of our knowledge, studies on the relationship between fentanyl and miR-204 in PTC cells are lacking. Results of qRT-PCR demonstrated that miR-204 expression was increased following 5 and $50 \mathrm{ng} / \mathrm{ml}$ fentanyl treatment (Figure 2(a)). Concentrations of fentanyl $>5 \mathrm{ng} / \mathrm{ml}$ exhibited 

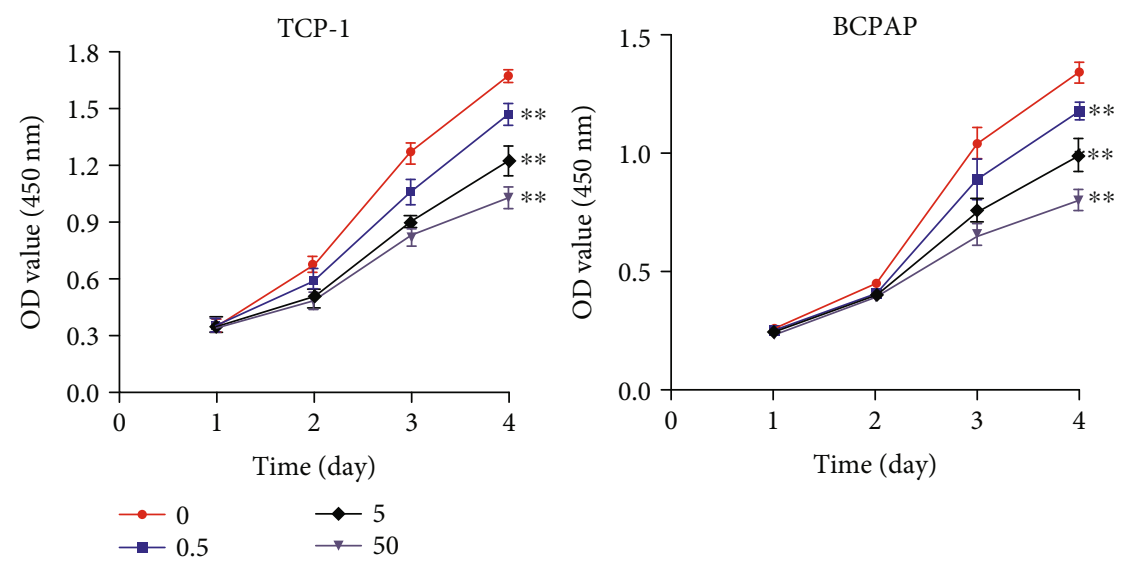

(a)
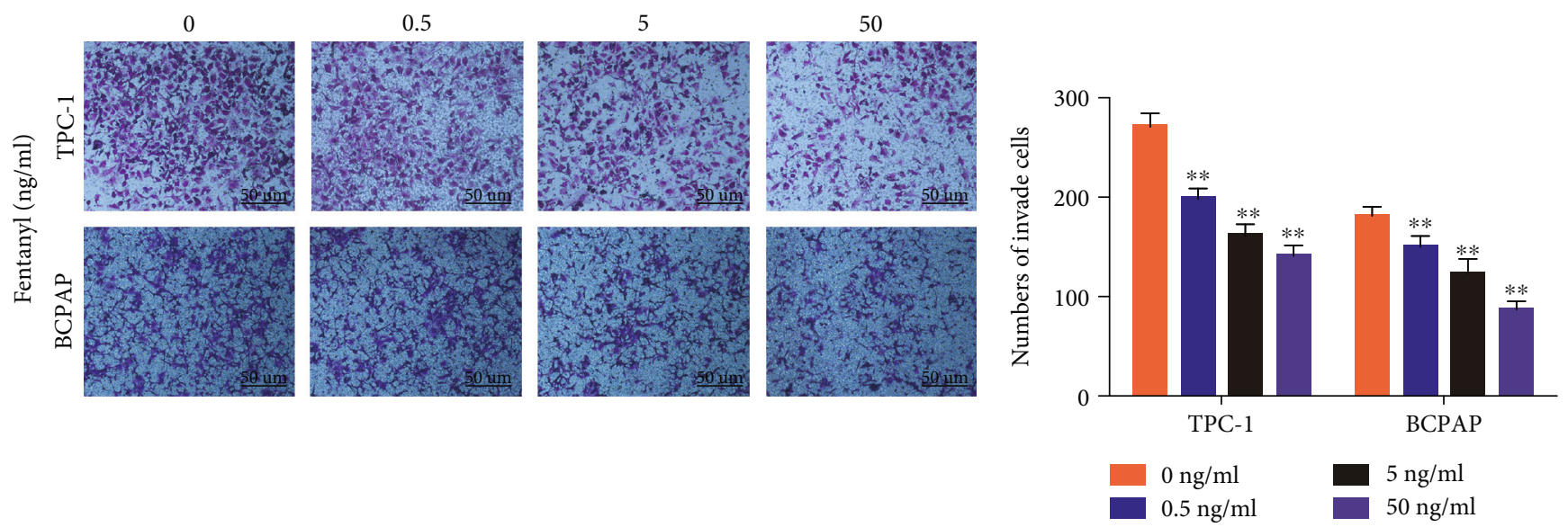

(b)

(c)

FIgURE 1: Fentanyl reduced the viability and invasion of PTC cells. (a) The results of CCK- 8 assays indicated that fentanyl reduced the cell viability at a dosage- and time-dependent manner. Repeated measurement ANOVA with Bonferroni corrections was used for statistical analysis. (b, c) The results of Transwell invasion assays confirmed that fentanyl significantly inhibited the invasion of PTC cells. One-way ANOVA was used for statistical analysis. Symbol of ${ }^{* *}$ indicates $P<0.05$ compared with PTC cells without fentanyl stimulation.

a remarkable inhibitory promotion on miR-204-5p expressions in PTC cells, and thus, a concentration of $5 \mathrm{ng} / \mathrm{ml}$ of fentanyl was chosen for further investigations. We utilized rescue experiments to understand the role of miR-204 in fentanyl-induced anticancer effects on PTC cells. CCK-8 assays and Transwell invasion assays showed that miR-204 inhibition by its specific inhibitor facilitated TCP-1 and BCPAP cell viability and invasion, which was reversed by following fentanyl treatment (Figures 2(b)-2(d)). These results reveal that miR-204 is involved in fentanyl-induced antitumor effects on PTC.

3.3. KLF5 Was the Target of miR-204. We used miRNA target prediction websites, such as starBase and TargetScan, to ascertain underlying mechanisms of miR-204 functioning in PTC cells and analyze miR-204 candidate targets. On the basis of these bioinformatics analyses, we chose a wellknown tumor promoter named KLF5 for further studies. The results of qRT-PCR and western blot analysis revealed a decrease in mRNA and protein levels of KLF5 in PTC cells transfected with miR-204 mimic (Figures 3(a) and 3(b)). Then, we found a complementary match between the seed sequence of miR-204 and the $3^{\prime}$ UTR region of KLF5 using the starBase (Figure 3(c)). Further studies were employed to explore whether KLF5 was directly targeted by miR-204. Luciferase reporter assays showed that miR-204 mimics led to inhibitory effects on the luciferase activity of the reporter with the wide-type sequence of KLF5, whereas no significant effect was observed in the mutant group (Figure 3(d)). To further verify the relationship between miR-204 and KLF5, we performed rescue experiments. As the results of CCK- 8 assays exhibited, KLF5 knockdown reduced TCP-1 and BCPAP cell viability and reversed the promotion of cell viability induced by the miR-204 inhibitor (Figure 3(e)). As expected, the results of Transwell invasion assays implied that the KLF5 knockdown inhibited TCP-1 and BCPAP cell invasion, which was negated by the miR-204 inhibitor (Figures 3(f) and 3(g)). These findings reveal that miR-204 modulates PTC cell growth by directly targeting KLF5.

3.4. Fentanyl Inhibited PTC Cell Viability and Invasion via the miR-204/KLF5 Axis. In this part, we were interested in the hypothesis that fentanyl may inhibit PTC development via the miR-204/KLF5 axis. For this purpose, more 

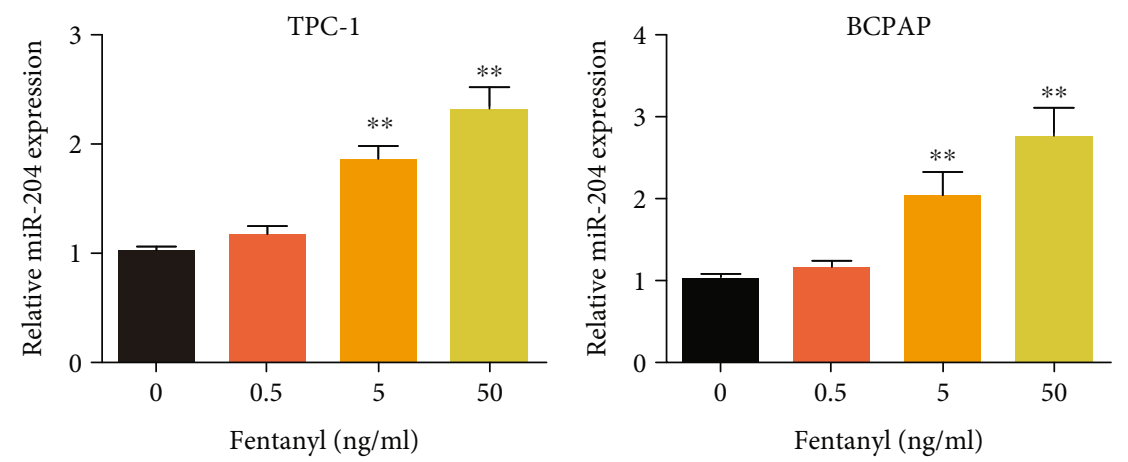

(a)
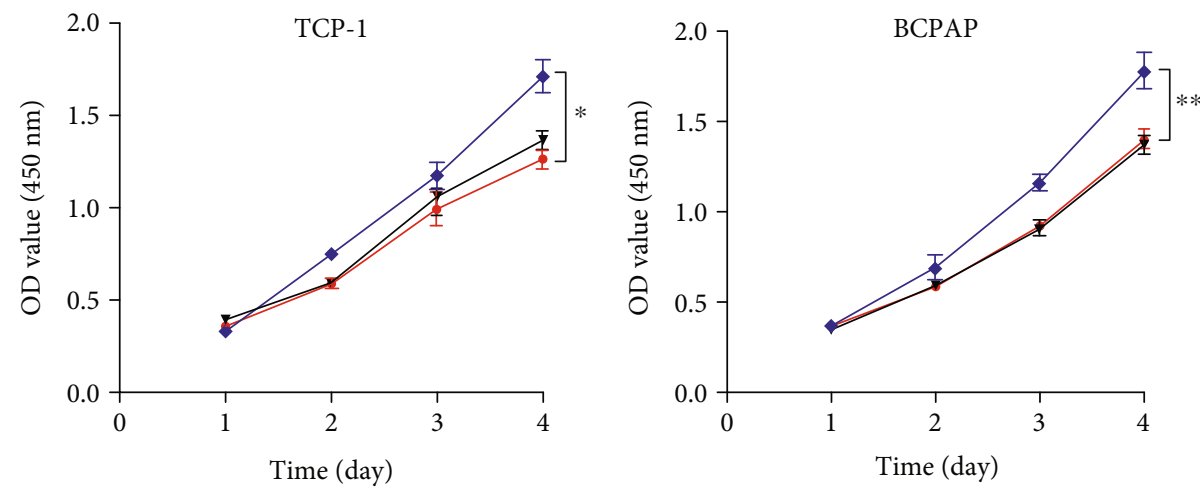

$\longrightarrow$ NC inhibitor+control
$\longrightarrow$ miR inhibitor+control
$\rightarrow$ miR inhibitor+fentanyl

(b)

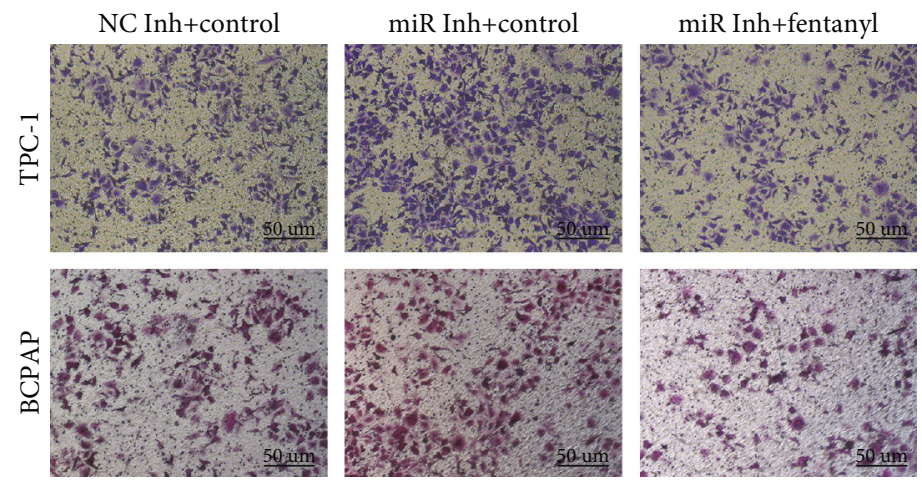

(c)

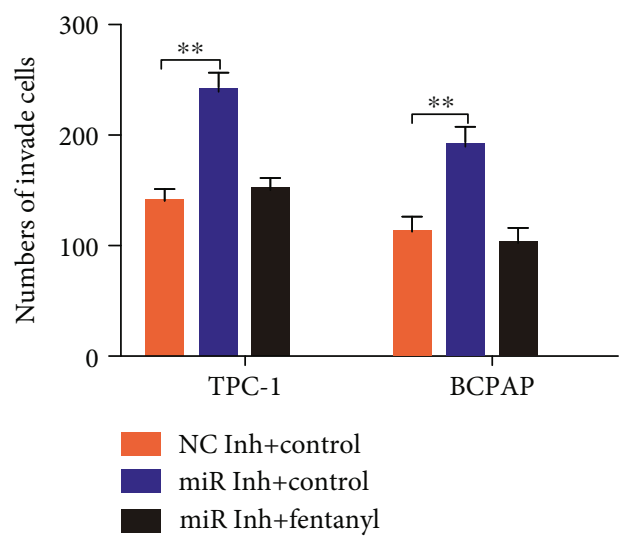

(d)

FIGURE 2: Fentanyl reversed the PTC cell viability and invasion via upregulation of miR-204. (a) The expression of miR-204 was increased by fentanyl treatment in a concentration-dependent manner $(>5 \mathrm{ng} / \mathrm{ml})$. (b) CCK- 8 assays showed that downregulation of miR-204 reversed the suppression of cell viability caused by fentanyl. (c, d) Transwell assays demonstrated that miR-204 downregulation antagonized the antiinvasion effects induced by fentanyl. Repeated measurement ANOVA with Bonferroni corrections was used for statistical analysis in (b) and one-way ANOVA was used in other panels. ${ }^{* *} P<0.05$.

experiments were used to verify the relationship among fentanyl, miR-204, and KLF5. The CCK-8 analysis of the present study showed that KLF5 overexpression reversed the fentanyl-mediated suppression of PTC viability (Figure 4(a)). The results of Transwell assays demonstrated that the restoration of KLF5 attenuates the enhancement of the cell invasion induced by fentanyl treatment (Figures 4(b) and 4(c)). Furthermore, our data showed that fentanyl inhibited the expression of KLF5, while this inhibition was reversed using the miR-204 inhibitor in PTC cells 


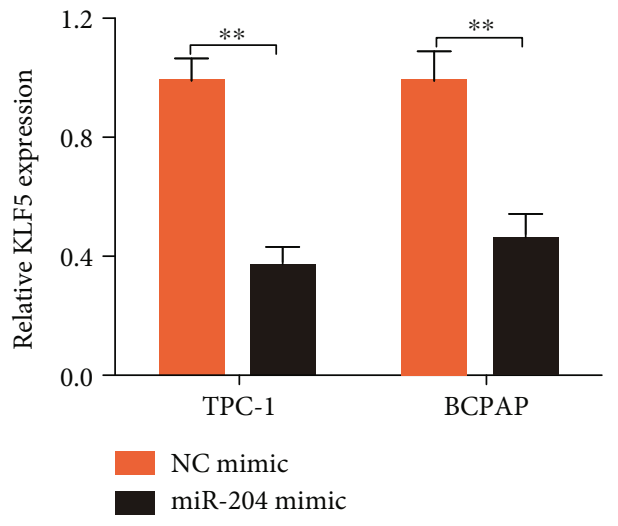

(a)

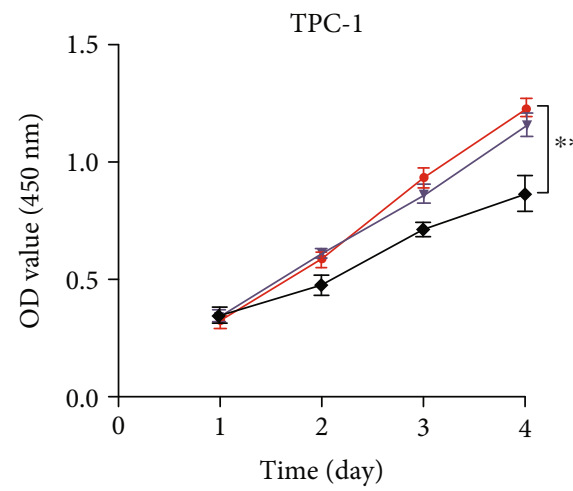

$\rightarrow$ NC inhibitor+si-NC

$\rightarrow$ miR inhibitor+si-KLF5

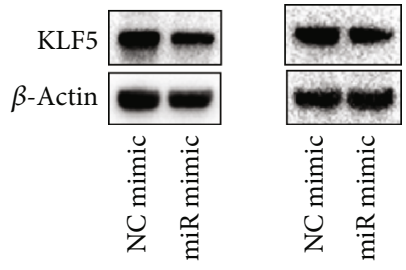

(b)

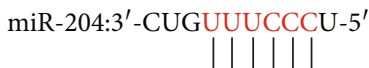

KLF5:5'-CCAAAAGGGU-3'

(c)

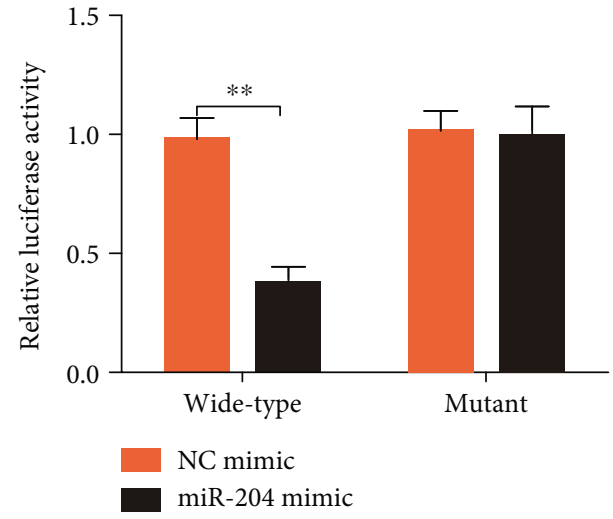

(d)

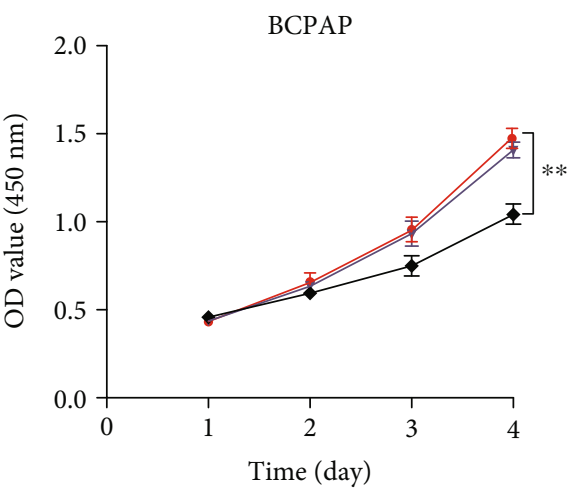

(e)

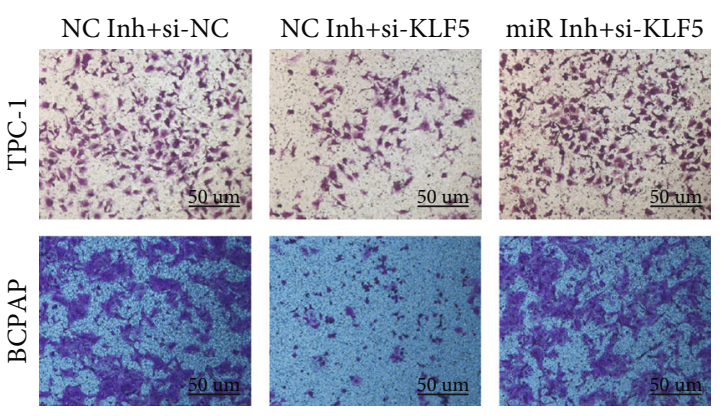

(f)

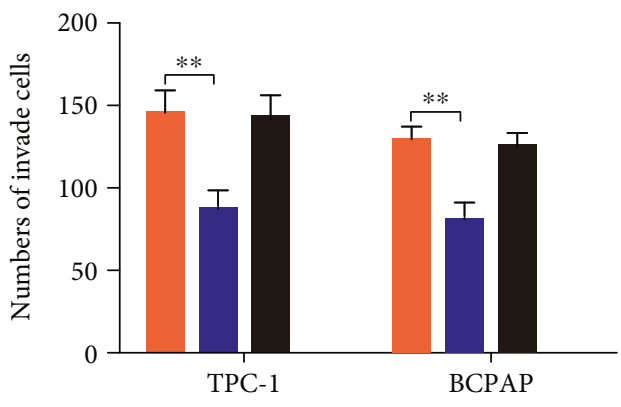

$\mathrm{NC}$ Inh+si-NC

NC Inh+si-KLF5

miR Inh+si-KLF5

(g)

FIgURe 3: KLF5 was the target of miR-204. (a) The results of PCR showed that miR-204 overexpression inhibited the expression of KLF5 at mRNA levels. (b) The results of western blot assays also verified the miR-204 overexpression at protein levels. (c) The sequence of $3^{\prime} \mathrm{UTR}$ of KLF5 contained the potential binding sites for miR-204. (d) The luciferase reporter assay identified that miR-204 directly targeted KLF5. (e) The results of CCK- 8 assays indicated that KLF5 knockdown reversed the positive effects on the proliferation of PTC cells induced by miR204 inhibitor. (f, g) The results of Transwell showed that miR-204 showed its negative effects on the cell invasion via targeting KLF5. Student's $t$-test was used for statistical analysis in ( $\mathrm{a}, \mathrm{d})$. Repeated measurement ANOVA with Bonferroni corrections was used in (e). One-way ANOVA was used in $(\mathrm{e}, \mathrm{g}) .{ }^{* *} \mathrm{P}<0.05$. 

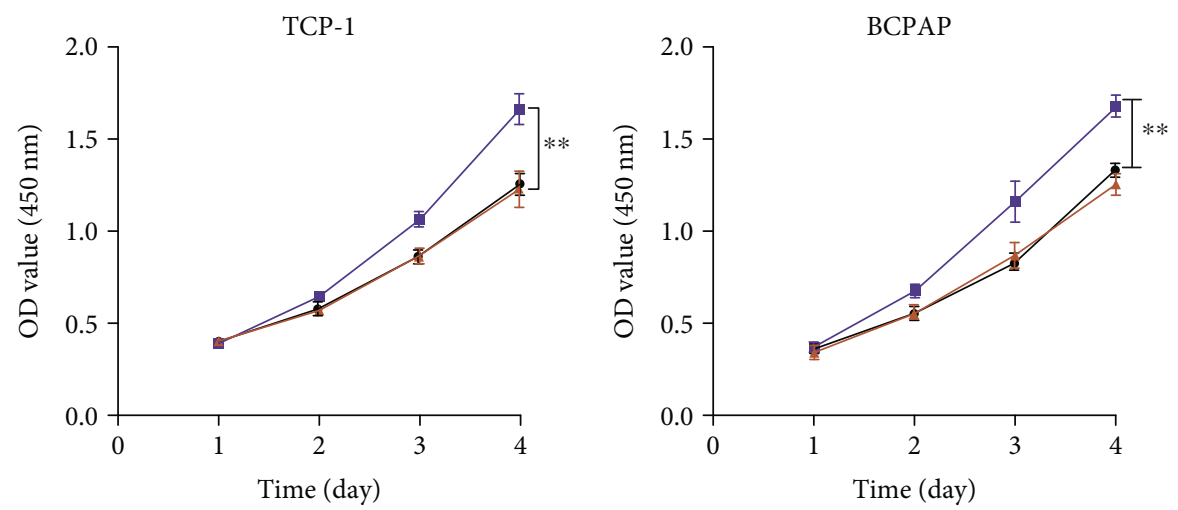

$\rightarrow$ NC+control
$\rightarrow$ KLF5+control
$\rightarrow$ KLF5+fentanyl

(a)

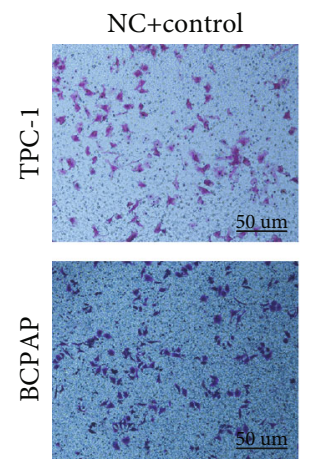

KLF5+control

KLF5+fentanyl
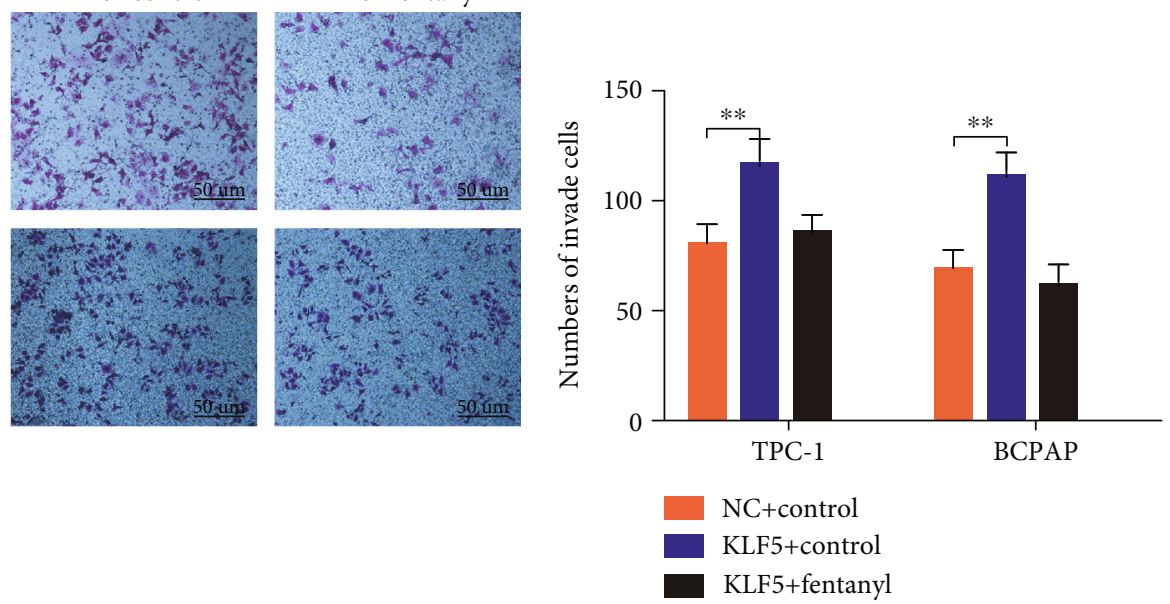

(b)

(c)

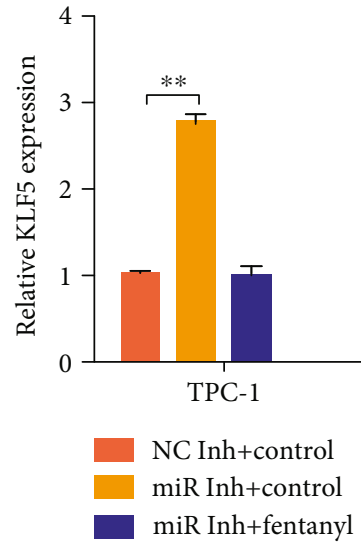

(d)

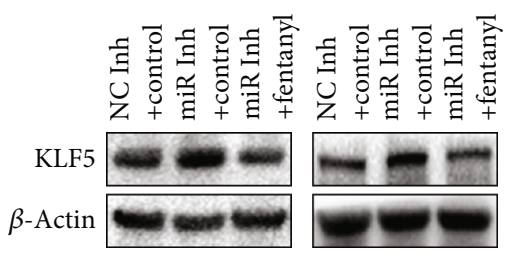

(e)

FIgURE 4: Fentanyl inhibited the cell viability and invasion via miR-204/KLF5 axis. (a) The CCK-8 assays were employed to explore the cell viability of PTC under fentanyl or KLF5 overexpression treatments. (b, c) The Transwell assays were used to test the cell invasion via fentanyl or KLF5 treatment. (d, e) The PCR and western blot assays were implied to detect the expressions of KLF5 under fentanyl or miR-204 inhibitor treatment. Repeated measurement ANOVA with Bonferroni corrections was used for statistical analysis in (a), and one-way ANOVA was used in other panels. ${ }^{* *} P<0.05$. 
(Figures 4(d) and 4(e)). Thus, these results indicated that fentanyl exerts its repressive effects on the cell viability and invasion via the miR-204/KLF5 axis.

\section{Discussion}

To date, surgery remains the predominant treatment for PTC but the distant metastasis is still the challenge of PTC patients [4]. Further investigations on underlying mechanisms of PTC tumorigenesis and metastasis are urgently required. With the advancement of anesthesia techniques, recent studies mainly focus on potential biological roles of anesthetics in tumor development. For instance, propofol showed its antitumor activity in pancreatic cancer cells by affecting miR34a-mediated LOC285194 and E-cadherin signals [19]. Fentanyl is a commonly used as an anesthesia that exerts its antitumor effects in many human cancers. For example, fentanyl was also demonstrated to inhibit the progression of human gastric cancer [20]. Celik and Duran provided evidence that fentanyl could reduce pancreatic cancer cell proliferation and cancer stem cell differentiation [21]. Zhang et al. [22] reported that fentanyl inhibited cell proliferation and invasion of colorectal cancer cells. A previous study demonstrated that propofol resulted in upregulation of miR-320a concomitant with reduction of HMGB1 and thus suppressed cell malignant behavior in PTC [23]. However, investigations on biological functions of fentanyl in PTC tumor progression are limited.

Wang et al. [7] reported that fentanyl induces the expression of miR-302b and subsequently decreases the cell invasive and proliferative abilities of ESCC. This study suggests that fentanyl partially exerts its functions by affecting miRNA expressions. Accounting studies demonstrated that misregulated miRNAs work as tumor suppressors or promoters participating in the pathogenesis of many cancers [24]. Several researchers focused on the role of miRNAs in tumorigenesis and tumor progression and found that miR-204, an important tumor-suppressive miRNA, repressed the expression of oncogene targets and thus suppressed the growth and metastasis of cancer cells in many tumors, such as colorectal cancer, endometrial carcinoma, and breast cancer [25-27]. A previous study proved that miR-204 is downregulated in PTC cells and tissues. The results of functional experiments indicated that overexpression of miR-204 inhibits the invasion and migration of PTC cells [17]. We identified in this work that miR-204 expression was upregulated under fentanyl treatment in PTC cells and fentanyl weakened the capability of PTC cells to proliferate and invade via repression of miR-204. Therefore, our data revealed that fentanyl inhibited the viability and invasion of PTC cells by enhancing miR-204 expression. Subsequent experiments verified that KLF5 is the target of miR-204 and involved in the mechanism of antitumor role of fentanyl in PTC.

KLF5 is a zinc-finger transcription factor closely correlated with different functions in tumor development, such as cell proliferation, apoptosis, adhesion, and invasion [28]. However, the role of KLF5 in various cancers is conflicting because it serves as a tumor promoter or suppressor. Ma et al. [29] showed that KLF5 reduced STAT3 activity and tumor metastasis in prostate cancer. Sun et al. [30] reported that liver cancer cells with KLF5 knockdown displays a sharp and round shape and promotes mesenchymal-to-epithelial transition. Our experiments demonstrated that KLF5 is the target of miR-204 and functional assays revealed that KLF5 overexpression promoted PTC cell viability and invasion of PTC. Poyil et al. [18] investigated the expression of KLF5 protein in a large cohort of PTC patient samples and explored its functional role and mechanism in PTC cell lines and PTC xenograft in mouse. They found that KLF5 overexpression was observed in $65.1 \%$ of all PTC cases, and it was significantly associated with aggressive clinicopathological parameters and poor outcome. However, they failed to perform further discussion concerning the mechanism of KLF5 overexpression in PTC. Fortunately, in our study, mechanistic analyses manifested that fentanyl inhibits the cell viability and invasion via the miR-204/KLF5 axis.

\section{Conclusion}

In summary, our work verified the antitumor effects of fentanyl in PTC through constant stimulation that reduces cell invasive and proliferative abilities of PTC cells. Further mechanistic analyses revealed that fentanyl performs its tumor-suppressive functions by enhancing miR-204 and reducing KLF5 in PTC cells. However, further investigations are required to strengthen the involvement of miR-204 and KLF5 in the mechanism of antitumor role of fentanyl in PTC. For instance, whether fentanyl inhibited the expression of KLF5 in PTC cells in a dose-dependent manner needs to be explored. Human sample and animal experiment are warranted to support clinical translation of the important role of fentanyl in treating PTC.

\section{Data Availability}

The data used to support the findings of this study are included within the article.

\section{Conflicts of Interest}

All authors declare that they have no conflict of interest.

\section{Acknowledgments}

This work was supported by Jiangsu Province " 333 " project (BRA2018020).

\section{References}

[1] H. Lim, S. S. Devesa, J. A. Sosa, D. Check, and C. M. Kitahara, "Trends in thyroid cancer incidence and mortality in the United States, 1974-2013," JAMA, vol. 317, no. 13, pp. 13381348, 2017.

[2] T. Carling and R. Udelsman, "Thyroid cancer," Annual Review of Medicine, vol. 65, no. 1, pp. 125-137, 2014.

[3] H. G. Vuong, N. P. Long, N. H. Anh et al., "Papillary thyroid carcinoma with tall cell features is as aggressive as tall cell variant: a meta-analysis," Endocrine Connections, vol. 7, no. 12, pp. R286-R293, 2018. 
[4] R. L. Siegel, K. D. Miller, and A. Jemal, "Cancer statistics, 2020," CA: a Cancer Journal for Clinicians, vol. 70, no. 1, pp. 7-30, 2020.

[5] T. K. Nuckols, L. Anderson, I. Popescu et al., "Opioid prescribing: a systematic review and critical appraisal of guidelines for chronic pain," Annals of Internal Medicine, vol. 160, no. 1, pp. 38-47, 2014.

[6] S. Mercadante, "Fentanyl buccal tablet for the treatment of cancer-related breakthrough pain," Expert Review of Clinical Pharmacology, vol. 8, no. 1, pp. 9-13, 2015.

[7] N. Wang, Z. Zhang, and J. Lv, "Fentanyl inhibits proliferation and invasion via enhancing miR-302b expression in esophageal squamous cell carcinoma," Oncology Letters, vol. 16, no. 1, pp. 459-466, 2018.

[8] C. Li, Y. Qin, Y. Zhong et al., "Fentanyl inhibits the progression of gastric cancer through the suppression of MMP-9 via the PI3K/Akt signaling pathway," Annals of Translational Medicine, vol. 8, no. 4, p. 118, 2020.

[9] R. C. Lee and V. Ambros, "An extensive class of small RNAs in Caenorhabditis elegans," Science, vol. 294, no. 5543, pp. 862864, 2001.

[10] Z. Wang, Y. Li, A. Ahmad et al., “Targeting miRNAs involved in cancer stem cell and EMT regulation: an emerging concept in overcoming drug resistance," Drug Resistance Updates, vol. 13, no. 4-5, pp. 109-118, 2010.

[11] G. Nie, H. Duan, X. Li et al., "MicroRNA205 promotes the tumorigenesis of nasopharyngeal carcinoma through targeting tumor protein p53-inducible nuclear protein 1," Molecular Medicine Reports, vol. 12, no. 4, pp. 5715-5722, 2015.

[12] J. Wu, L. Yin, N. Jiang et al., "MiR-145, a microRNA targeting ADAM17, inhibits the invasion and migration of nasopharyngeal carcinoma cells," Experimental Cell Research, vol. 338, no. 2, pp. 232-238, 2015.

[13] Y. Zhou, L. Yin, H. Li, L. H. Liu, and T. Xiao, "The LncRNA LINC00963 facilitates osteosarcoma proliferation and invasion by suppressing miR-204-3p/FN1 axis," Cancer Biology \& Ther$a p y$, vol. 20, no. 8, pp. 1141-1148, 2019.

[14] J. Zhang, L. Xing, H. Xu et al., "miR-204-5p suppress lymph node metastasis via regulating CXCL12 and CXCR4 in gastric cancer," Journal of Cancer, vol. 11, no. 11, pp. 3199-3206, 2020.

[15] Y. Liang, E. Li, H. Zhang, L. Zhang, Y. Tang, and Y. Wanyan, "Silencing of lncRNA UCA1 curbs proliferation and accelerates apoptosis by repressing SIRT1 signals by targeting miR204 in pediatric AML," Journal of biochemical and molecular toxicology, vol. 34, no. 3, article e22435, 2020.

[16] K. Santiago, Y. Chen Wongworawat, and S. Khan, "Differential microRNA-signatures in thyroid cancer subtypes," Journal of Oncology, vol. 2020, Article ID 2052396, 14 pages, 2020.

[17] L. Liu, J. Wang, X. Li et al., "MiR-204-5p suppresses cell proliferation by inhibiting IGFBP5 in papillary thyroid carcinoma," Biochemical and Biophysical Research Communications, vol. 457, no. 4, pp. 621-626, 2015.

[18] P. Pratheeshkumar, A. K. Siraj, S. P. Divya et al., "Prognostic value and function of KLF5 in papillary thyroid cancer," Cancers (Basel), vol. 13, no. 2, p. 185, 2021.

[19] H. Wang, H. Jiao, Z. Jiang, and R. Chen, "Propofol inhibits migration and induces apoptosis of pancreatic cancer PANC1 cells through miR-34a-mediated E-cadherin and LOC285194 signals," Bioengineered, vol. 11, no. 1, pp. 510$521,2020$.
[20] Y. Qin, L. Li, J. Chen et al., "Fentanyl inhibits progression of human gastric cancer MGC-803 cells by NF- $\kappa$ B downregulation and PTEN upregulation in vitro," Oncology Research, vol. 20, no. 2, pp. 61-69, 2012.

[21] F. Celik and T. Duran, "Effects of fentanyl on pancreatic cancer cell proliferation and cancer stem cell differentiation," Cellular and Molecular Biology (Noisy-le-Grand, France), vol. 65, no. 7, pp. 21-25, 2019.

[22] X. L. Zhang, M. L. Chen, and S. L. Zhou, "Fentanyl inhibits proliferation and invasion of colorectal cancer via $\beta$-catenin," International Journal of Clinical and Experimental Pathology, vol. 8, no. 1, pp. 227-235, 2015.

[23] M. Li, L. Qu, F. Chen, and X. Zhu, "Propofol upregulates miR320a and reduces HMGB1 by downregulating ANRIL to inhibit PTC cell malignant behaviors," Pathology-Research and Practice, vol. 216, no. 4, article 152856, 2020.

[24] D. F. Dai, Y. W. Tan, L. F. Guo, A. F. Tang, and Y. S. Zhao, "Identification of exosomal miRNA biomarkers for diagnosis of papillary thyroid cancer by small RNA sequencing," European Journal of Endocrinology, vol. 182, no. 1, pp. 111-121, 2020.

[25] C. C. Huang, C. H. Hung, T. W. Hung, Y. C. Lin, C. J. Wang, and S. H. Kao, "Dietary delphinidin inhibits human colorectal cancer metastasis associating with upregulation of miR-204-3p and suppression of the integrin/FAK axis," Scientific reports, vol. 9, no. 1, article 18954, 2019.

[26] T. K. Chung, T. S. Lau, T. H. Cheung et al., "Dysregulation of microRNA-204 mediates migration and invasion of endometrial cancer by regulating FOXC1," International Journal of Cancer, vol. 130, no. 5, pp. 1036-1045, 2012.

[27] J. Zeng, G. Li, Y. Xia et al., "miR-204/COX5A axis contributes to invasion and chemotherapy resistance in estrogen receptorpositive breast cancers," Cancer Letters, vol. 492, pp. 185-196, 2020.

[28] R. Liu, P. G. Shi, Z. M. Zhou et al., "Krüpple-like factor 5 is essential for mammary gland development and tumorigenesis," The Journal of Pathology, vol. 246, no. 4, pp. 497-507, 2018.

[29] J. B. Ma, J. Y. Bai, H. B. Zhang et al., "KLF5 inhibits STAT3 activity and tumor metastasis in prostate cancer by suppressing IGF1 transcription cooperatively with HDAC1," Cell death \& disease, vol. 11, no. 6, p. 466, 2020.

[30] L. Zhang, Y. Wu, J. Wu et al., "KLF5-mediated COX2 upregulation contributes to tumorigenesis driven by PTEN deficiency," Cellular Signalling, vol. 75, article 109767, 2020. 